\title{
Statistical characterization of evaluation strategies for fringe projection systems by means of a model-based noise prediction
}

\author{
Marc Fischer, Marcus Petz, and Rainer Tutsch \\ Institut für Produktionsmesstechnik, Technische Universität Braunschweig, Braunschweig, Germany \\ Correspondence to: Marc Fischer (marc.fischer@tu-bs.de)
}

Received: 28 September 2016 - Revised: 9 February 2017 - Accepted: 26 February 2017 - Published: 6 April 2017

\begin{abstract}
For optical 3-D measurement systems, camera noise is the dominant uncertainty factor when optically cooperative surfaces are measured in a stable and controlled environment. In industrial applications repeated measurements are seldom executed for this kind of measurement system. This leads to statistically suboptimal results in subsequent evaluation steps as the important information about the quality of individual measurement points is lost. In this work it will be shown that this information can be recovered for phase measuring optical systems with a model-based noise prediction. The capability of this approach will be demonstrated exemplarily for a fringe projection system and it will be shown that this method is indeed able to generate an individual estimate for the spatial stochastic deviations resulting from image sensor noise for each measurement point. This provides a valuable tool for a statistical characterization and comparison of different evaluation strategies, which is demonstrated exemplarily for two different triangulation procedures.
\end{abstract}

\section{Introduction}

For optical 3-D measurement systems, like fringe projection systems, the achievable accuracy mainly depends on the environmental conditions and the properties of the measurement object. In a production-related environment, systematic deviations caused for example by temperature fluctuations or vibrations are the dominant factors. In contrast, a wellcontrolled environment leads to a strong influence of the surface properties of the object under test. While the deviations are correlated with the micro-topography in the case of noncooperative surfaces, stochastic deviations caused by camera noise come to the fore in the case of cooperative surfaces. Depending on the local lighting conditions, these can differ by up to an order of magnitude for different measurement points on the object.

In industrial applications of optical 3-D measurement systems, the measurements are usually not repeated for estimating repeatability. The deviations of the measurement process may be characterized by measurements of spherical and planar artefacts in the context of acceptance tests and reverifications according to VDI 2634 (VDI, 2012). It has to be pointed out however that this is not to be confused with a measurement uncertainty as it is only a method to ensure that the system is working in conformity with the specifications. The experimental determination of a task-specific measurement uncertainty, for example according to VDA5 (VDA, 2011), is only worth the effort in the case of a small measurement object portfolio and large lot sizes. In addition this method characterizes the whole measurement process only in a general way. As an alternative, the use of "virtual fringe projection systems" (Haskamp et al., 2012) is being discussed as a tool for task-specific error analysis. Although this approach can be seen as state of the art for tactile coordinate measuring machines (Trenk et al., 2004), in the case of fringe projection systems it is not yet established in the industry.

The missing assessment of the quality of each individual measurement point leads to statistically suboptimal results in subsequent evaluation steps as for example this information is not available as a weighting factor for the matching of geometric primitives. Because of the use case outlined above, i.e. the measurement of cooperative surfaces in a wellcontrolled environment, the geometric deviations of the mea- 


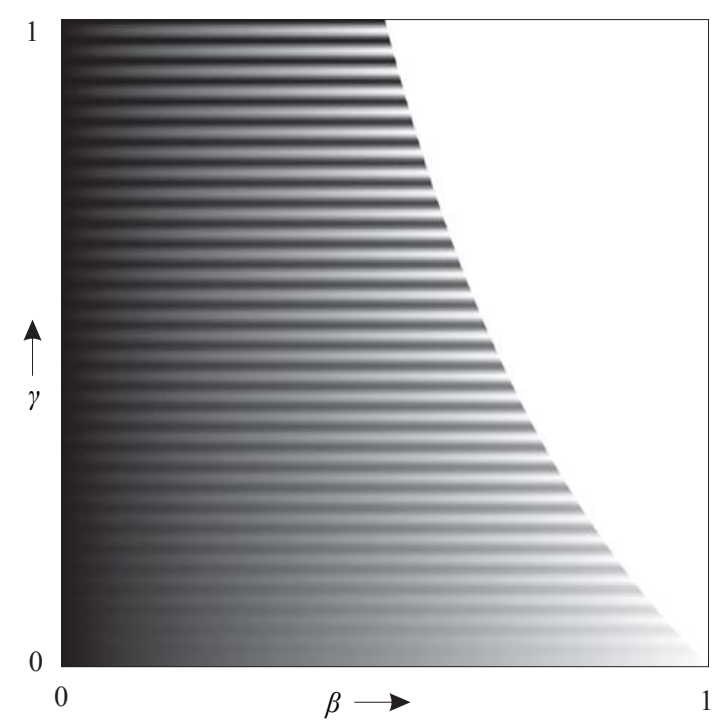

Figure 1. Fringe pattern with locally varying visibility $\gamma$ and illumination $\beta$. In the right part of the diagram, points that do not meet the constraint for the maximum illumination (clipping) are painted white.

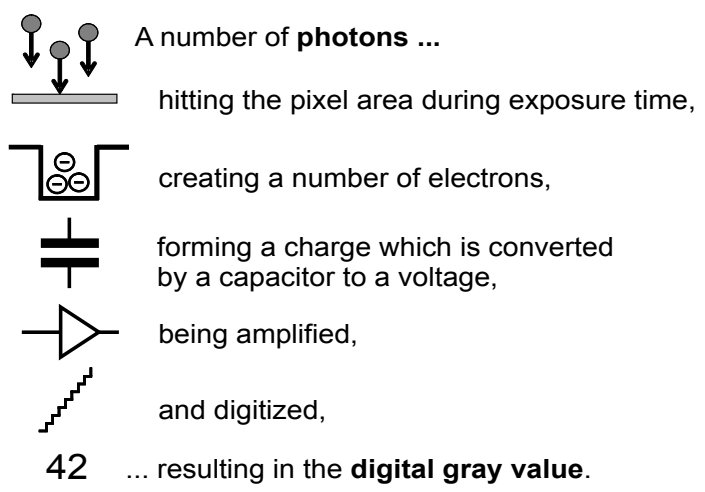

Figure 2. Physical model of signal generation in a CCD-camera pixel according to EMVA 1288 (EMVA, 2010).

sured points are dominated by camera noise, and they can be estimated directly from the measurement data if the noise characteristic of the measurement process is modelled. This approach will be described in the following sections and is a first important step towards a task-specific uncertainty estimation for this kind of measurement system.

\section{Optical measurement systems with structured illumination}

Optical measurement systems that use intensity patterns to spatially encode an object are referred to as "structured illumination" techniques. Among others, a widely applied coding approach is based on multiple phase-shifted sinusoidal fringe patterns. In this case the actual coding takes place in the time domain, as the phase-shifted patterns are usu-

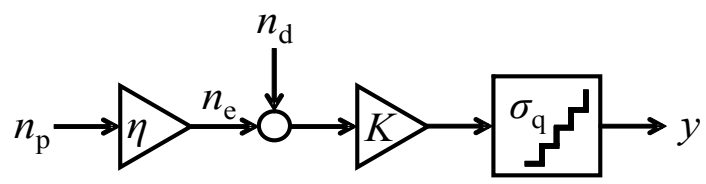

Figure 3. Mathematical model for the signal generation according to EMVA 1288 (EMVA, 2010).

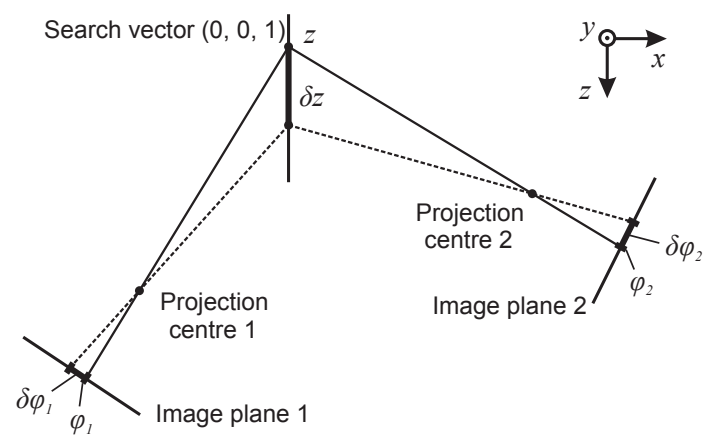

Figure 4. Propagation of phase deviations into the object space in a fringe projection system with two cameras. The implementation is based on a passive triangulation along a search vector in the $z$ direction.

ally recorded sequentially. The recorded intensity $I_{i}$ for each pixel in the $i$ th phase-shifted image can be written as

$I_{i}=I_{\mathrm{sat}} \beta\left[1+\gamma \cos \left(\varphi+\psi_{i}\right)\right] \quad i=1, \ldots, M$,

where $I_{\mathrm{sat}}$ is the saturation intensity of the pixel, $\beta$ the relative unmodulated intensity (illumination) and $\gamma$ the relative fringe contrast (visibility), $\varphi$ the unknown phase angle, $\psi_{i}$ the applied phase shift and $M$ the number of phase-shifted images. The parameters $\beta$ and $\gamma$ describe the local lighting conditions for a given pixel independent of the camera. Both are in the range of [0...1] with an additional constraint for the maximum of $\beta$ for a given $\gamma$ because $I_{i}$ should always be smaller than $I_{\text {sat }}$. The effect of $\beta$ and $\gamma$ on the recorded images can be visualized by means of a fringe pattern with locally varying visibility and illumination as demonstrated in Fig. 1. As can be seen, the optimal fringe contrast can be achieved for $\beta=0.5$ and $\gamma=1$.

With the recorded intensities $I_{i}$ according to Eq. (1) the corresponding unknown phase value can be calculated for each pixel by means of phase-shift algorithms. A class of widely applied algorithms is based on evenly spaced phase shifts over one period ("symmetric $M$-step algorithms"), with the famous four-step algorithm for $M=4$ :

$\tan \varphi=\frac{I_{3}-I_{1}}{I_{4}-I_{2}} \psi_{i}=\left(\frac{\pi}{2} ; \pi ; \frac{3 \pi}{2} ; 2 \pi\right)$.

\section{Modelling and prediction of phase noise}

For symmetric $M$-step algorithms a simple relation between the absolute unmodulated intensity $I^{\prime}$, the intensity noise of 


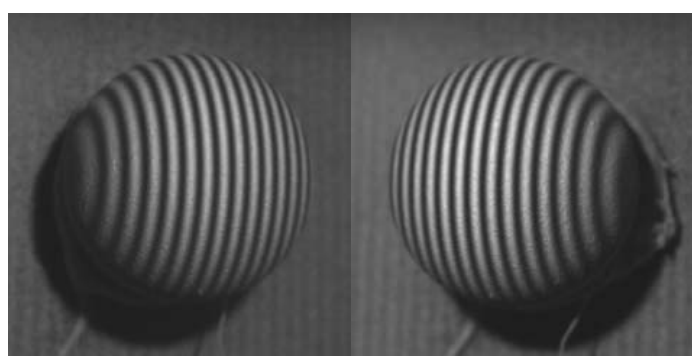

Figure 5. Cropped camera views of the measured sphere with the fringe pattern projected onto it.

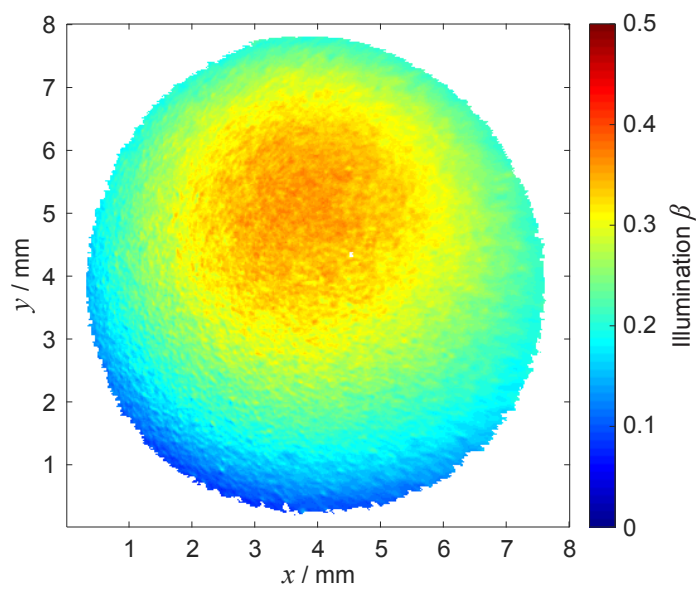

Figure 6. Visualization of the distribution of parameter $\beta$ across the measured ball bearing sphere as recorded by camera 1 .

the image sensor $\sigma_{I}$ and the phase noise $\sigma_{\varphi}$ can be found according to Brophy (1990), Rathjen (1995) and Surrel (1997) as

$\sigma_{\varphi}=\sqrt{\frac{2}{M}} \frac{1}{\gamma} \frac{\sigma_{I}}{I^{\prime}}=\sqrt{\frac{2}{M}} \frac{1}{\gamma} \frac{1}{\mathrm{SNR}}$.

Although this expression is based on the assumption of signal-independent Gaussian noise $\left(\sigma_{I}=\right.$ const.), where the signal-to-noise ratio (SNR) would increase linearly with the unmodulated intensity $I^{\prime}$, in Fischer et al. (2012) it has been shown that it is also valid for a more complex expression of SNR resulting from the advanced linear camera model of the EMVA 1288 guideline (EMVA, 2010). Furthermore it has been demonstrated in Fischer et al. (2012) and in more detail in Fischer (2016) that Eq. (3) can be developed into a prediction method that is able to estimate the phase noise directly from the measured intensities. The basic physical model of signal generation in a camera pixel is shown in Fig. 2, and the resulting mathematical model in Fig. 3.

It can be seen that the fundamental process is a conversion, first from the number of photons $n_{\mathrm{p}}$ to the number of electrons $n_{\mathrm{e}}$ with the total quantum efficiency $\eta$ as the conversion factor and second to digital grey values $y$ with the system gain $K$ as the conversion factor. Three noise sources

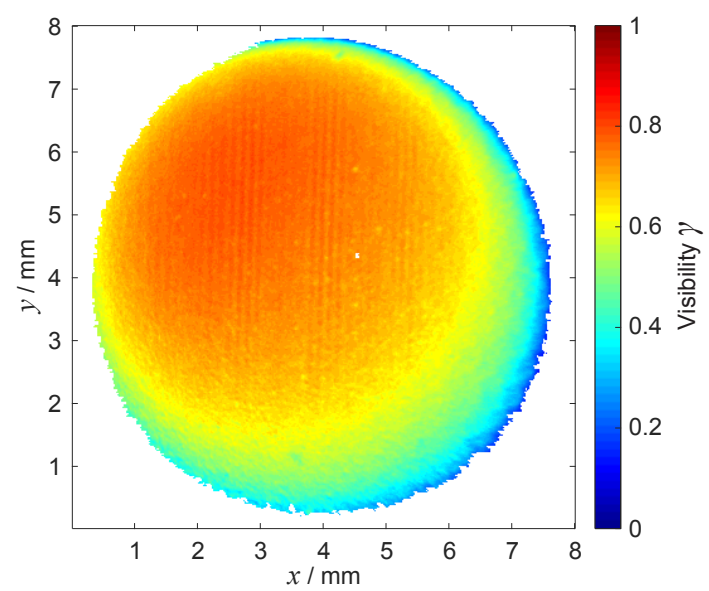

Figure 7. Visualization of the distribution of parameter $\gamma$ across the measured ball bearing sphere as recorded by camera 1 .

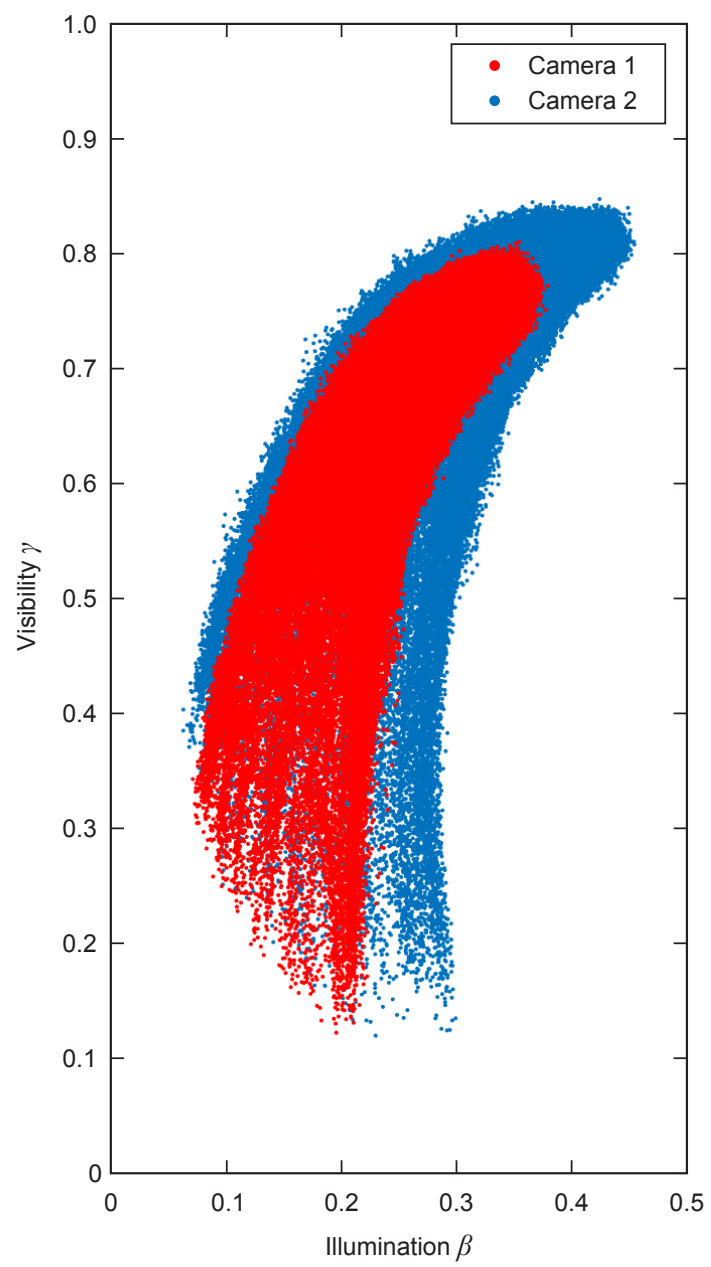

Figure 8. Achieved coverage of the $\beta$ - and $\gamma$-parameter space of both cameras available for the experiments. This can be considered typical of a fringe projection measurement of an optically cooperative surface. 


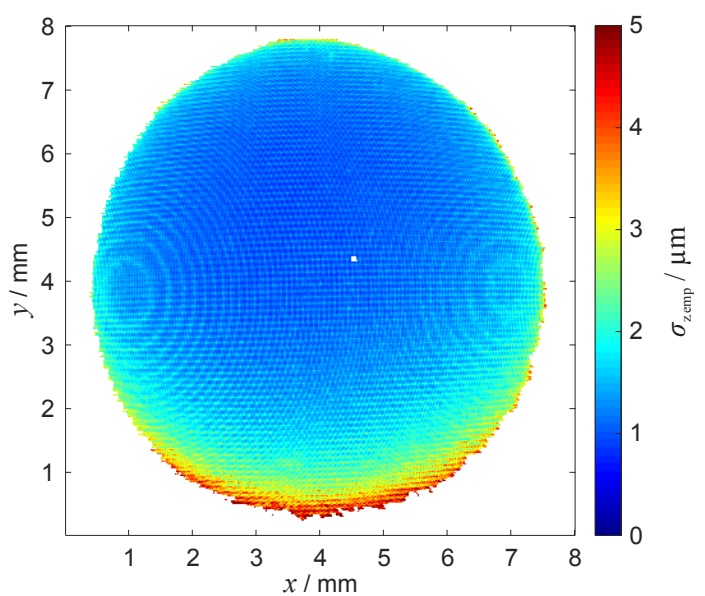

Figure 9. Empirically determined reference data for the stochastic deviations of the measured $z$ coordinate $\sigma_{z \text {.emp }}$.

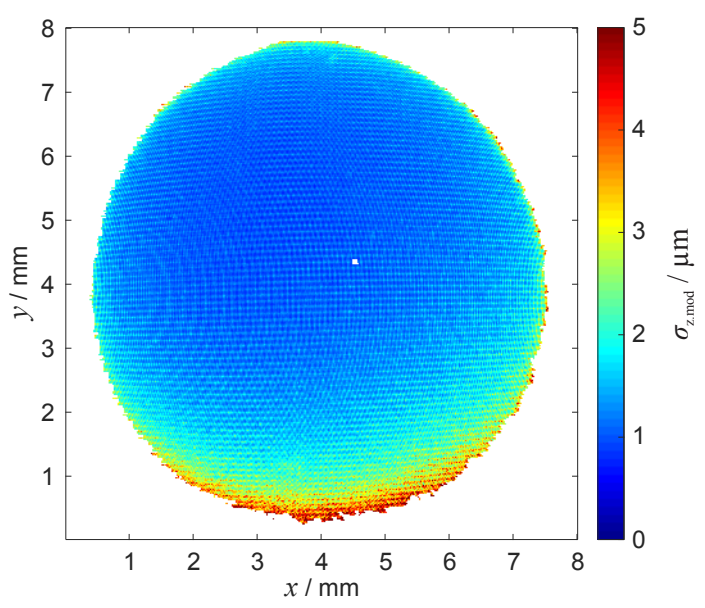

Figure 10. Estimated value according to the noise model $\sigma_{z . \bmod }$ corresponding to Fig. 9.

are then added to the model with the following assumptions: the number of electrons $n_{\mathrm{e}}$ is Poisson-distributed $\left(\sigma_{\mathrm{e}}^{2}=\mu_{\mathrm{e}}\right)$, the number of dark noise electrons $n_{\mathrm{d}}$ is normally distributed $\left(\mu_{\mathrm{d}} ; \sigma_{\mathrm{d}}\right)$, and the quantization noise is uniformly distributed in $[-1 / 2,1 / 2]$; thus, $\sigma_{\mathrm{q}}^{2}=1 / 12$. It should be noted that the photon noise in the model subsumes two physical effects, as the number of photons $n_{\mathrm{p}}$ emitted from the light source is already a Poisson-distributed quantity. Additionally the detection of the photons and the generation of the corresponding number of electrons $n_{\mathrm{e}}$ on the image sensor is a Poisson process. Thus the resulting distribution, as the convolution of both effects, is still a Poisson distribution. Therefore the amount of photon noise in the image only depends on the mean intensity of the observed light, and it can be completely characterized by the camera model depicted in Fig. 3, without an additional consideration of the light source.

For the four-step algorithm a simple relation between the recorded intensities $y_{i}$ and the estimated phase noise $\sigma_{\varphi \text { mod }}$

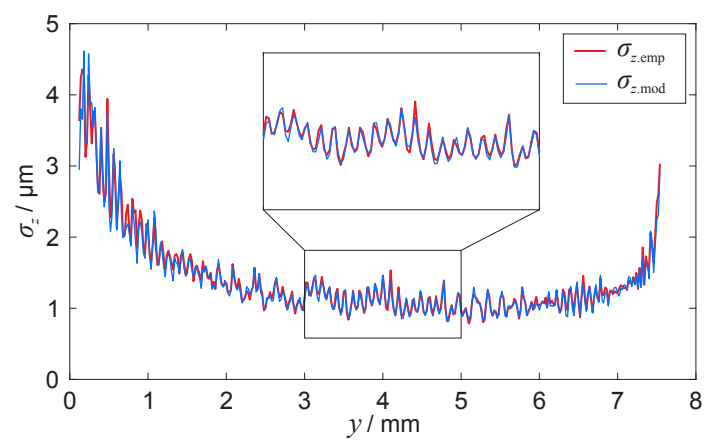

Figure 11. Plot of a single data column of $\sigma_{z \text {.emp }}$ and $\sigma_{z \text {.mod }}$ across the pole of the sphere.

can be derived based on this camera model (Fischer et al., 2012):

$\sigma_{\varphi \cdot \bmod }=\sqrt{\frac{\frac{K}{2}\left(y_{1}+y_{2}+y_{3}+y_{4}\right)+N_{4}}{\left(y_{1}-y_{3}\right)^{2}+\left(y_{2}-y_{4}\right)^{2}}}$,

with the combined noise constant $N_{4}$ as

$N_{4}=2 K^{2}\left(\sigma_{\mathrm{d}}^{2}-\mu_{\mathrm{d}}\right)+\frac{1}{6}$.

In the following it will be shown how the general phase-noise estimation can be applied to a given measurement system and further developed into a prediction method for the stochastic coordinate deviations of the measured surface points. In Sects. 4 and 5 this propagation of the phase noise into the object space is described exemplarily for two different implementations. As the focus of this paper is the prediction of spatial noise caused by camera noise, which is the dominant effect in optical measurements on cooperative surfaces in a well-controlled environment, systematic effects have been neglected in the noise model. To emphasize this, the term "quality metric" instead of "uncertainty" is used when referring to this prediction in the following sections.

\section{Example 1: passive triangulation along a search vector}

The propagation of the stochastic phase deviations into the object space depends on the actual implementation of the triangulation strategy. In this first example a passive triangulation along a search vector has been implemented, where "passive" in this context means that the projection is only used for the optical coding of the surface, whereas the actual triangulation is done with two cameras. In this example an iterative method is used, where each object point is found on a regular $x y$ grid along a search vector in the $z$ direction of a local workpiece coordinate system by minimizing the observed phase differences $\delta \varphi_{1}$ and $\delta \varphi_{2}$ in both camera views. The basic geometry of this process is depicted in Fig. 4; a detailed description of the actual implementation and the experimental validation can be found in the following sections. 


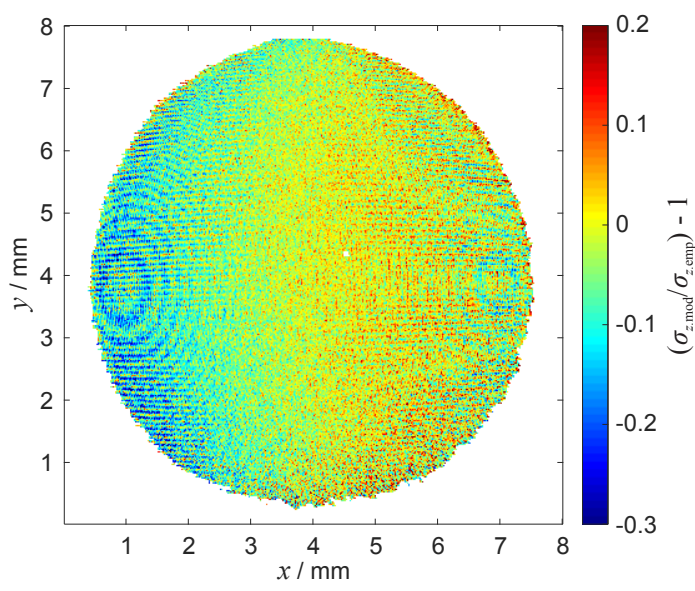

Figure 12. Relative deviation of the estimated value $\sigma_{z \text {.mod }}$ from the empirically determined reference value $\sigma_{z \text {.emp }}$.

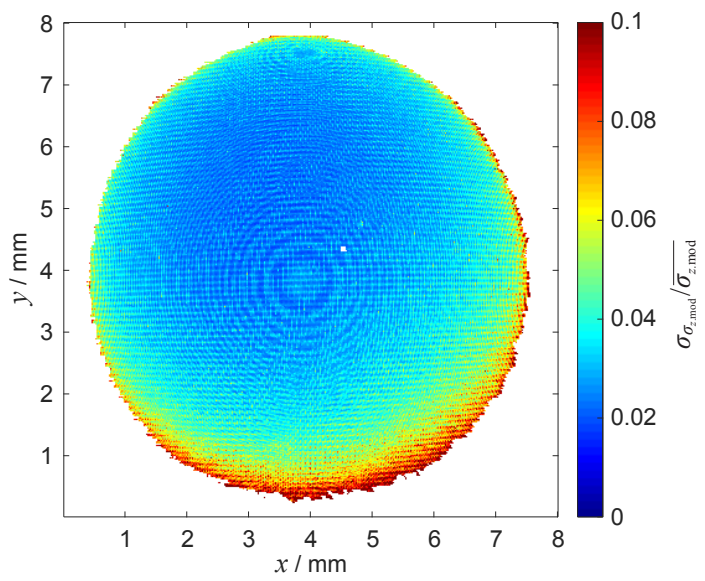

Figure 13. Relative stochastic deviation (precision) of the estimated value $\sigma_{z \text {.mod. }}$.

\subsection{Heterodyne phase evaluation}

The spatial coding of the surface via fringe projection is realized by a combination of phase shifting and heterodyne evaluation. The whole image sequence consists of $12 \mathrm{im}$ ages: three slightly different fringe widths $\lambda_{1}, \lambda_{2}, \lambda_{3}$ with four phase shifts of $\pi / 2$ each. For each valid measurement point this yields a phase value $\varphi_{c, p, w}$ per camera index $c$, pixel index $p$ and fringe width index $w$, and in addition an estimation for the phase noise $\sigma_{\varphi \text {.mod }}$ according to Eq. (4) as a quality metric $u_{c, p, w}$ :

$u_{c, p, w}=\sigma_{\varphi \cdot \bmod }(c, p, w)$.

For each pixel in both cameras the three independent phase measurements have to be normalized to a reference fringe width $\lambda_{\text {ref }}=\lambda_{1}$ and averaged with the variance of the spatial coding $\left(\lambda_{w} \cdot u_{c, p, w}\right)^{2}$ as an inverse weighting factor. This results in the combined phase value $\varphi_{c, p}$ and the combined

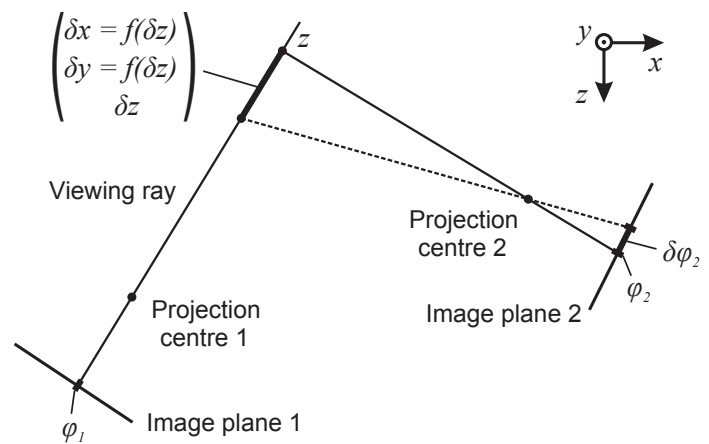

Figure 14. Propagation of phase deviations into the object space in a fringe projection system with two cameras. The implementation is based on a passive triangulation along a viewing ray of camera 1 .

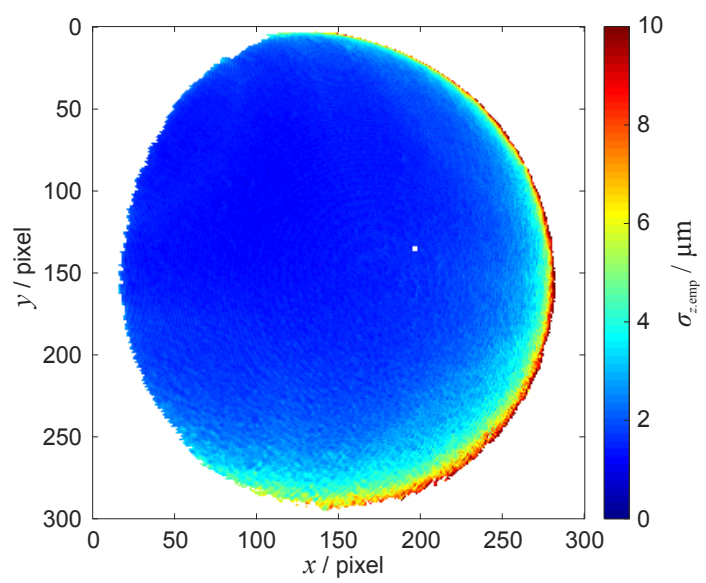

Figure 15. Empirically determined reference data for the stochastic deviations of the measured $z$ coordinate $\sigma_{z \text {.emp }}$.

quality metric $u_{c, p}$ :

$\varphi_{c, p}=\frac{\sum_{w=1}^{3} \frac{\varphi_{c, p, w} \cdot \frac{\lambda w}{\lambda_{\mathrm{ref}}}}{\left(\lambda_{w} \cdot u_{c, p, w}\right)^{2}}}{\sum_{w=1}^{3} \frac{1}{\left(\lambda_{w} \cdot u_{c, p, w}\right)^{2}}}$

and

$u_{c, p}=\frac{1}{\lambda_{\text {ref }}} \sqrt{\frac{1}{\sum_{w=1}^{3} \frac{1}{\left(\lambda_{w} \cdot u_{c, p, w}\right)^{2}}}}$.

\subsection{Subpixel interpolation}

Because the image coordinates are calculated with subpixel resolution by means of a resection into the image plane, the corresponding phase values have to be interpolated. In the case of a bilinear interpolation that has been applied here, the interpolated phase value $\varphi_{c}$ can be calculated from the phase 
values $\varphi_{c, 1} \ldots \varphi_{c, 4}$ of the four surrounding pixels $p=1 \ldots 4$ by a weighted summation with the corresponding subareas $a_{c, p}$ as weighting factors:

$\varphi_{c}=\sum_{p=1}^{4} \varphi_{c, p} a_{c, p}$ with $\sum_{p=1}^{4} a_{c, p}=1$.

The influence of this bilinear interpolation on the quality metric can be incorporated as follows:

$u_{c}=\sqrt{\sum_{p=1}^{4}\left(u_{c, p} a_{c, p}\right)^{2}}$.

\subsection{Triangulation}

For the propagation of the resulting estimated phase noise in both camera views $u_{1}$ and $u_{2}$ into the object space, the triangulation principle depicted in Fig. 4 results in additional coupling terms $C_{12}$ and $C_{21}$ because of the applied minimization of squared phase deviations. These coupling terms depend on the partial derivative of the object coordinate $z$ with respect to the phase angles $\varphi_{1}$ and $\varphi_{2}$. The influence of the imaging geometry is completely described by the local values of these sensitivity coefficients. The structure of the coupling terms results from the characteristic of the applied iterative solving algorithm that a change in $\delta \varphi_{1}$ varies $z$ and consequently $\delta \varphi_{2}$ and vice versa:

$C_{12}=\frac{\left(\frac{\delta z}{\delta \varphi_{2}}\right)^{2}}{\left(\frac{\delta z}{\delta \varphi_{1}}\right)^{2}+\left(\frac{\delta z}{\delta \varphi_{2}}\right)^{2}}$

and

$C_{21}=\frac{\left(\frac{\delta z}{\delta \varphi_{1}}\right)^{2}}{\left(\frac{\delta z}{\delta \varphi_{1}}\right)^{2}+\left(\frac{\delta z}{\delta \varphi_{2}}\right)^{2}}$.

With these coupling terms the resulting standard deviation of the estimated spatial noise $\sigma_{z \text {.mod }}$ for a passive triangulation along a search vector in the $z$ direction can finally be calculated as

$\sigma_{z . \bmod }=\sqrt{\left(u_{1} \frac{\delta z}{\delta \varphi_{1}} C_{12}\right)^{2}+\left(u_{2} \frac{\delta z}{\delta \varphi_{2}} C_{21}\right)^{2}}$.

\subsection{Experimental set-up and methodology}

For the experimental validation a photogrammetric fringe projection system has been used, composed of two cameras with a resolution of $1024 \times 768$ pixel and a digital projector with a resolution of $800 \times 600$ pixel. The measurement volume is a cuboid with a size of about $20 \mathrm{~mm} \times 15 \mathrm{~mm} \times 10 \mathrm{~mm}$. As the object under test a ballbearing sphere with a nominal diameter of $8 \mathrm{~mm}$ has been chosen. It has been prepared with an optically cooperative surface by spraying with titanium dioxide. In Fig. 5 the views of both cameras onto the prepared sphere are shown. Because only the validity of the noise model is under investigation, the geometric quality of the sphere, i.e. form deviations and roughness, is not relevant in these experiments. A sphere has been chosen because the curvature of the surface leads to a good coverage of the $\beta$ - and $\gamma$-parameter space in one single measurement. The variation of the surface normal from the pole of the sphere to the Equator results in a decrease in $\beta$, whereas the large aperture and the focusing onto the pole lead to a small depth of focus and a decreasing $\gamma$ respectively. In Figs. 6 and 7 the distribution of both parameters across the sphere has been visualized exemplarily for camera 1 .

The resulting parameter space of combinations of $\beta$ and $\gamma$ that is available for the experimental validation of the noise model is shown in Fig. 8 for both cameras. The difference between the distributions for the two cameras could be traced back to a slightly different aperture setting and a small off-centre position of the sphere. The parameter space covered by these experiments can be considered typical of a measurement of optically cooperative surfaces with a fringe projection system. A significantly different distribution is to be expected when the measurement object is made out of a volume-scattering material for example. In this case the maximum visibility $\gamma_{\max }$ would be decreased, which in turn would typically lead to an increased illumination $\beta$ as the exposure time could be adapted accordingly.

In addition to the object point $z$ coordinate itself, which is found by the iterative triangulation process, as described above, Eq. (13) yields the corresponding estimated stochastic deviations $\sigma_{z \text {.mod. }}$. The $x$ and $y$ coordinates compose a regular grid with a spacing of $20 \mu \mathrm{m}$ in the conducted experiments. The evaluation is based on a series of 800 repeated measurements of the sphere, lasting for about $2.5 \mathrm{~h}$. The subsequent analysis has been conducted only with measurement points that are valid across the whole measurement series, which has been the case for a total of 103449 points.

\subsection{Experimental results}

It has been deduced from the data that thermal influences lead to varying inner and outer orientation of the cameras over the course of the measurement series, resulting in an observed movement of the point cloud. For the centroid of the point cloud, a mean displacement in the $z$ direction with a range of about $160 \mathrm{~nm}$ and a standard deviation of about $27 \mathrm{~nm}$ can be observed, which is not evenly distributed across the sphere. This influence superimposes the empirical data shown in the following sections and slightly affects the quality of the comparison between the empirically determined and estimated spatial noise as the standard deviation for points with a high quality is about $1 \mu \mathrm{m}$.

From the whole series of 800 measurements, the empirical standard deviation of the $z$ coordinate $\sigma_{z \text {.emp }}$ has been 


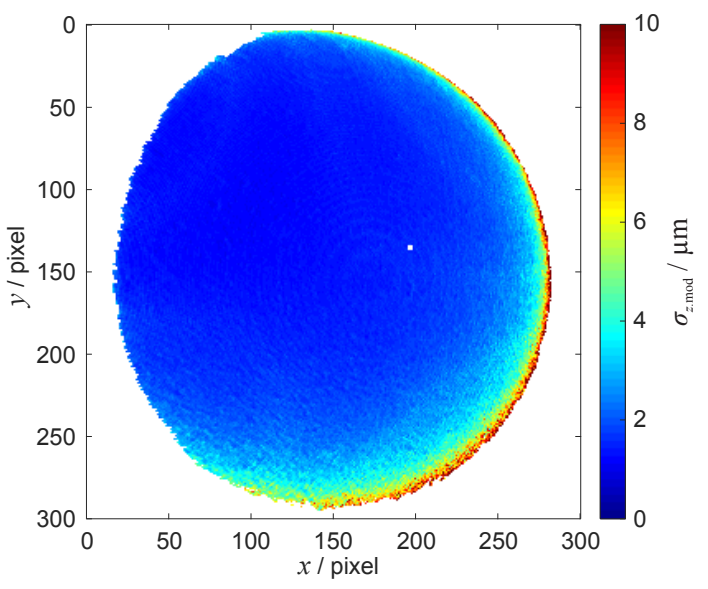

Figure 16. Estimated value according to the noise model $\sigma_{z \text {.mod }}$ corresponding to Fig. 15.

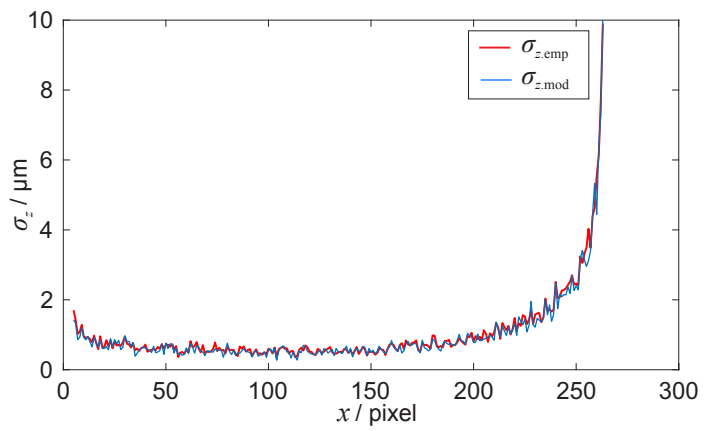

Figure 17. Plot of a single data row of $\sigma_{z \text {.emp }}$ and $\sigma_{z \text {.mod }}$ across the pole of the sphere.

calculated. The results are shown in Fig. 9. Points measured with good lighting conditions that are near the pole of the sphere feature a spatial noise of the order of $1 \mu \mathrm{m}$, whereas this value increases to more than $5 \mu \mathrm{m}$ towards the boundary of the point cloud. For the validation this reference value has to be compared to the estimated spatial noise $\sigma_{z \text {.mod }}$ calculated from a single measurement of the series according to the noise model of Eq. (13). The results are shown in Fig. 10, and it can be seen that this is indeed consistent with Fig. 9. The very good agreement between empirical and estimated spatial noise can be better assessed in Fig. 11, where a column of the original data across the pole of the sphere is shown. It can be seen that both the low-frequency variations and the high-frequency interpolation effects are correctly estimated by the model.

For a better assessment of the prediction quality over the whole measured geometry, Fig. 12 shows the relative deviation of the estimated value $\sigma_{z \text {.mod }}$ from the empirically determined reference value $\sigma_{z \text {.emp }}$. There is a prominent lowfrequency structure, with the left side of the sphere having negative deviations on average, whereas the right side more often features positive deviations. This could be traced back

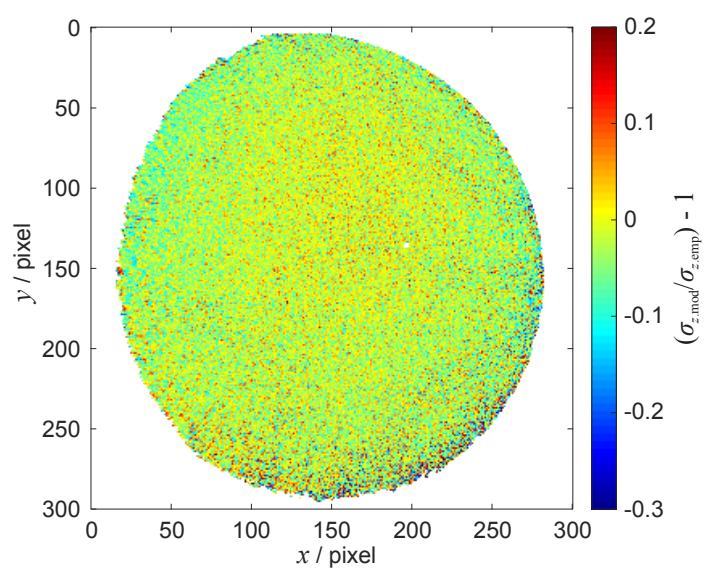

Figure 18. Relative deviation of the estimated value $\sigma_{z . \bmod }$ from the empirically determined reference value $\sigma_{z \text {.emp. }}$

to the thermal influence discussed above. The circular highfrequency structures on the left and on the right side are caused by interpolation effects. They are visible in the empirical data (Fig. 9) and the estimated values (Fig. 10) as well but with different amplitude, leading to the observed deviations. The reason for these differences could not be found and this topic is under further investigation.

The relative deviation of the estimation is $-3.48 \%$ on average with a standard deviation of $7.09 \%$. The negative mean value implies that the estimated stochastic deviation is slightly smaller than the reference value, which is plausible as there are other influence factors besides camera noise. Furthermore it has to be taken into account that a non-negligible part of the deviations is caused by the statistical properties of the reference itself. The confidence interval for the empirical calculation of a standard deviation with $n=800$ and $k=2$ is about $\pm 5 \%$ and of the same order of magnitude as the observed deviations.

The precision of the estimation method can be characterized by the distribution of $\sigma_{z \text {.mod }}$ across all measurements of the series. The resulting distribution of the relative stochas-

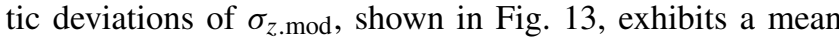
value of $3.61 \%$, a standard deviation of $1.52 \%$ and maximum values of about $10 \%$. These stochastic deviations also contribute to the observed deviations from the reference values discussed above. They are strongly correlated with the expected spatial noise for the corresponding measurement point, with the points near the pole $\left(\sigma_{z} \approx 1 \mu \mathrm{m}\right)$ showing typical deviations of about $2 \%$, whereas in the boundaries of the point cloud $\left(\sigma_{z} \approx 5 \mu \mathrm{m}\right)$ this value increases to about $10 \%$.

\section{Example 2: passive triangulation along a viewing ray}

Similarly to the first example discussed in Sect. 4, where object points are found on a regular grid, the triangulation along 


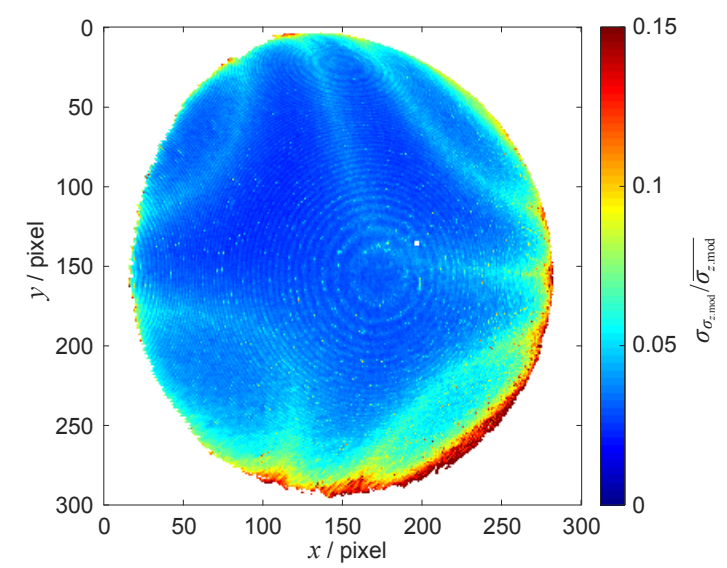

Figure 19. Relative stochastic deviation (precision) of the estimated value $\sigma_{z \text {.mod }}$.

a viewing ray is also a passive technique where the 3-D information is calculated from two camera views and the projector is only used as an optical surface coding device. Viewing rays in this context are the principal rays for the given imaging geometry from each pixel through the common projection centre of a pinhole camera model, including deviations from the ideal imaging geometry by means of distortion terms. In contrast to the triangulation along a vector in the $z$ direction, in this triangulation method the object point position is searched along these viewing rays of camera 1 until the spatial coding information corresponds to the view of camera 2, thus minimizing $\delta \varphi_{2}$. The geometry of this method is shown in Fig. 14.

\subsection{Heterodyne phase evaluation}

The spatial coding of the surface via fringe projection is realized with the same method as described in Sect. 4.1, i.e. by a combination of phase shifting and heterodyne evaluation with an image sequence of 12 images: three slightly different fringe widths with four $\pi / 2$ phase shifts each. Thus Eqs. (7) and (8) for the calculation of the combined phase value $\varphi_{c, p}$ and the combined quality metric $u_{c, p}$ are also valid in this example.

\subsection{Subpixel interpolation}

In the case of a viewing-ray-based triangulation method, there is no need for subpixel interpolation in the first camera view because the starting points of the viewing rays are usually defined on pixel centre coordinates. The phase value and quality metric for the first camera can then simply be calculated from these pixel values:

$\varphi_{1}=\varphi_{1, p}$ and $u_{1}=u_{1, p}$.

Alternatively the starting point for viewing rays could be defined as the corner of a pixel, incorporating information from all four surrounding pixels. Due to an averaging effect this would decrease spatial noise as well as the structure resolution of the measurement. In this case subpixel interpolation according to Eqs. (9) and (10) has to be taken into account for the first camera too. In the implementation considered in this example however this is only necessary for the second camera, where a corresponding image point is found by a resection into the image plane, leading to non-integer pixel coordinates in general.

\subsection{Triangulation}

As described above, the passive triangulation along a viewing ray uses the same iterative approach to find an object point, but in contrast to example 1 in this case it is bound to a specific viewing ray in the first camera. The point search is done by moving the object point along this viewing ray and minimizing the phase difference $\delta \varphi_{2}$ in the second camera view. For the first camera, the image coordinates remain fixed to specific integer values. Therefore the resulting standard deviation of the estimated spatial noise $\sigma_{z \text {.mod }}$ for a passive triangulation along a viewing ray of camera 1 only depends on the geometrical sensitivity of the second camera and can be calculated as

$\sigma_{z . \bmod }=\sqrt{\left(u_{1} \frac{\delta z}{\delta \varphi_{2}}\right)^{2}+\left(u_{2} \frac{\delta z}{\delta \varphi_{2}}\right)^{2}}=\frac{\delta z}{\delta \varphi_{2}} \sqrt{u_{1}^{2}+u_{2}^{2}}$.

\subsection{Experimental set-up and methodology}

The experimental set-up and methodology for the second example are the same as described in Sect. 4.4; in fact, the raw images of the identical measurement have been used for both implementations. In this case the viewing rays of the first camera define the measurement grid, which is non-regular in object space due to optical distortions and the geometry of the measurement object. The resulting distribution of the distance between adjacent object points has a median value of about $25 \mu \mathrm{m}$. The number of measurement points that are valid across the whole measurement series of 800 repeated measurements is 59925 in this case.

\subsection{Experimental results}

The experimental findings for this second example are in accordance with the findings of the first example described in Sect. 4.5, and the results are qualitatively equivalent. The empirical standard deviation of the $z$ coordinate $\sigma_{z \text {.emp }}$ is shown in Fig. 15 and the estimated spatial noise $\sigma_{z \text {.mod }}$ in Fig. 16. Here the spatial noise is slightly higher compared to example 1, which results from the difference in the interpolation schemes. As the starting point of each viewing ray of the first camera is the pixel centre point, there is no averaging over adjacent pixels. The large increase in $\sigma_{z}$ on the right side of the sphere is caused by the imaging geometry. On this side 
of the sphere the surface normal is nearly perpendicular to the viewing rays, resulting in an increased sensitivity $\delta z / \delta \varphi_{2}$. In Fig. 17 a row of the original data across the pole of the sphere is shown. It can be seen that the spatial noise is correctly estimated by the model. Figure 18 shows the relative deviation of the estimated value $\sigma_{z \text {.mod }}$ from the empirically determined reference value $\sigma_{z . \mathrm{emp}}$. It is $-1.98 \%$ on average, with a standard deviation of $5.49 \%$. The precision of the estimation method, shown in Fig. 19, exhibits a mean value of $4.36 \%$ and a standard deviation of $2.10 \%$. These values are comparable to the findings for the first example.

\section{Conclusion and summary}

In this work a model-based approach for the estimation of stochastic coordinate deviations, caused by camera noise in phase-measuring optical 3-D measurement systems, has been proposed. In the case of a fringe projection system measuring an optically cooperative surface in a well-controlled environment this influence is the dominating factor.

Starting from a general phase-noise estimation the propagation into the object space has been derived for two different triangulation methods. For both triangulation methods the noise estimation has been experimentally validated and the estimation quality, i.e. the precision and accuracy of the estimated values, has been assessed. The precision of this estimation is better than $10 \%$ for most of the measured points, which can be seen as feasible for practical applications, especially given the fact that such information is generally not available at all for most industrial applications. These estimated spatial noise values can be used for further data processing as a point quality metric. Although in this work the deviations have been simplified to scalar $z$ components for illustration purposes, their vectorial property has to be taken into account if these estimated deviations should be used as weighting factors in a sphere fit for example. In this case they have to be projected to radial deviations to yield meaningful values.

Data availability. The data presented in this paper have been generated by means of repeated measurements with a fringe projection system and consist of several hundred images of a ball-bearing sphere. The image series itself does not carry any scientifically relevant meaning besides demonstrating the prediction method described in this work. The experiments can easily be reproduced with other fringe projection systems. Therefore, the image series is not available online, but the authors will provide sample data upon request.

Competing interests. The authors declare that they have no conflict of interest.
Acknowledgements. This work is partly based on prior research that has been funded by the Deutsche Forschungsgemeinschaft (DFG) under grant Pe1402/21. The authors gratefully acknowledge the funding.

Edited by: U. Neuschaefer-Rube

Reviewed by: two anonymous referees

\section{References}

Brophy, C. P.: Effect of intensity error correlation on the computed phase of phase-shifting interferometry, JOSA A, 7, 537-541, doi:10.1364/JOSAA.7.000537, 1990.

EMVA: European Machine Vision Association, EMVA Standard 1288 - Standard for Characterization of Image Sensors and Cameras, Release 3.0, 29 November, 2010.

Fischer, M., Petz, M., and Tutsch, R.: Vorhersage des Phasenrauschens in optischen Messsystemen mit strukturierter Beleuchtung, Tech. Mess., 79, 451-458, doi:10.1524/teme.2012.0256, 2012.

Fischer, M.: Deflektometrie in Transmission - Ein neues Messverfahren zur Erfassung der Geometrie asphärischer refraktiver Optiken, Dissertation, Technische Universität Braunschweig, Schriftenreihe des Instituts für Produktionsmesstechnik, Band 12, Aachen: Shaker, ISBN 978-3844043211, 83-87, 2016.

Haskamp, K., Kästner, M., Ohrt, C., and Reithmeier, E.: Estimation of measurement uncertainties using virtual fringe projection technique, Appl. Opt., 51, 1516-1520, doi:10.1364/AO.51.001516, 2012.

Rathjen, C.: Statistical properties of phase-shift algorithms, JOSA A, 12, 1997-2008, doi:10.1364/JOSAA.12.001997, 1995.

Surrel, Y.: Additive noise effect in digital phase detection, Appl. Opt., 36, 271-276, doi:10.1364/AO.36.000271, 1997.

Trenk, M., Franke, M., and Schwenke, H.: The "Virtual CMM", a software tool for uncertainty evaluation-practical application in an accredited calibration lab, Proc. of ASPE: Uncertainty Analysis in Measurement and Design, 2014.

VDA: Qualitätsmanagement in der Automobilindustrie, Band 5: Prüfprozesseignung, Eignung von Messsystemen, Mess- und Prüfprozessen, Erweiterte Messunsicherheit, Konformitätsbewertung, 2. vollständige überarbeitete Auflage 2010, aktualisiert 2011, 2011.

VDI/VDE: 2634 Blatt 2:2012-8, Optische 3-D-Messsysteme Bildgebende Systeme mit flächenhafter Antastung, 2012. 